\title{
A new approach to characterize postural deficits in chemotherapy-induced peripheral neuropathy and to analyze postural adaptions after an exercise intervention
}

Sarah Kneis ${ }^{1 *}$ (D), Anja Wehrle², Daniela Dalin, Isabella Katharina Wiesmeier ${ }^{1}$, Johann Lambeck ${ }^{1}$, Albert Gollhofer ${ }^{3}$, Hartmut Bertz ${ }^{1}$ and Christoph Maurer ${ }^{1}$

\begin{abstract}
Background: Postural instability presents a common and disabling consequence of chemotherapy-induced peripheral neuropathy (CIPN). However, knowledge about postural behavior of CIPN patients is sparse. With this pilot study, we used a new approach to i) characterize postural impairments as compared to healthy subjects, ii) allocate possible abnormalities to a set of parameters describing sensorimotor function, and iii) evaluate the effects of a balance-based exercise intervention.

Methods: We analyzed spontaneous and externally perturbed postural control in eight CIPN patients before and after a balance-based exercise intervention by using a modification of an established postural control model. These findings were compared to 15 matched healthy subjects.
\end{abstract}

Results: Spontaneous sway amplitude and velocity were larger in CIPN patients compared to healthy subjects. CIPN patients' reactions to external perturbations were smaller compared to healthy subjects, indicating that patients favor vestibular over proprioceptive sensory information. The balance-based exercise intervention up-weighted proprioceptive information in patients.

Conclusions: CIPN patients' major postural deficit may relate to underuse of proprioceptive information that results in a less accurate posture control as spontaneous sway results indicate. The balance-based exercise intervention is able to partially correct for this abnormality. Our study contributes to a better understanding of postural impairments in CIPN patients and suggests an effective treatment strategy.

Trial registration: German Clinical Trials Register: DRKS00004340, retrospectively registered 04 January 2013.

Keywords: Postural stability, Chemotherapy-induced peripheral neuropathy, Motor control, Sensory weighting, Model

\footnotetext{
*Correspondence: sarah.kneis@uniklinik-freiburg.de

1 Department of Neurology and Neuroscience, Faculty of Medicine, Medical

Center, University of Freiburg, Freiburg, Germany

Full list of author information is available at the end of the article
}

(c) The Author(s). 2020 Open Access This article is distributed under the terms of the Creative Commons Attribution 4.0 International License (http://creativecommons.org/licenses/by/4.0/), which permits unrestricted use, distribution, and reproduction in any medium, provided you give appropriate credit to the original author(s) and the source, provide a link to the Creative Commons license, and indicate if changes were made. The Creative Commons Public Domain Dedication waiver (http://creativecommons.org/publicdomain/zero/1.0/) applies to the data made available in this article, unless otherwise stated. 


\section{Clinical message}

- CIPN patients present greater postural sway than healthy control subjects associated with postural instability

- CIPN patients use less proprioceptive information than control subjects entailing less accuracy for posture control

- A balance-based exercise intervention can partially correct for the proprioceptive underuse of CIPN patients

\section{Background}

Chemotherapy-induced peripheral neuropathy (CIPN) is a common and clinically relevant side-effect of cancer treatment [1-3]. CIPN can cause treatment delays and dose reductions, interfering with general outcome or compromising survival [3-6]. Consequences of CIPN can lead to excessive healthcare costs and resource use [7]. Symptoms of CIPN primarily include paraesthesia, dysesthesia, numbness and pain with a symmetric, distal, length-dependent "glove and stocking" distribution $[3,8]$ and limit patients' everyday life considerably. Additionally, CIPN patients often suffer from postural instability [9-17], contributing to a lower quality of life [5, 18], a higher risk of mortality [19-22] and increased healthcare costs $[23,24]$.

Quantitative reports about CIPN patients' postural instability are currently rising: CIPN has been associated with reduced gait abilities $[13,14]$ and changes in center of pressure (COP) displacements [10, 12, 25]. However, underlying mechanisms are sparsely described [10, 25]: Wampler et al. [10] assumed that besides somatosensory impairments also diminished vestibular function causes increased postural sway in CIPN patients. Furthermore, in an earlier study we found changes in elicitability and sensitivity of spinal reflex circuitry associated with postural instability in CIPN patients [25]. More comprehensive knowledge about neuropathy-induced postural instability has been derived from patients diagnosed with diabetes: Bonnet et al. [26] deduced larger COP displacements, which were more pronounced with visual disturbance. Diabetic neuropathy patients seem to delay postural reactions [27], shift from ankle to hip strategy [26, 28, 29], and seem to use vestibular rather than proprioceptive cues [30]. However, proprioception may be essential for stability in both quiet stance and during unexpected postural perturbations [26, 31-33], since it provides information about lower limb orientation with respect to the support base $[34,35]$. They report a clear increase in postural sway when proprioceptive cues are deficient $[10,26,32]$. Our first aim here is to characterize the postural deficits in CIPN and to extract the sensorimotor abnormalities using a well-established model of postural control [36-38].
Concerning treatment, knowledge about the management of CIPN-induced postural instability is still sparse [39-41]. Generally, it is increasingly suggested to focus on strength and especially balance exercises in order to improve physical functioning of CIPN patients [11], what we could confirm in a randomized controlled trial by ourselves [42]. Until recently, there are only two other interventional studies showing that balance exercises improved CIPN-related postural control deficits $[39,41]$. However, evidence from diabetes research on neuropathy further supports this assumption [43-45]. Balance training in general has proven to enhance postural stability by inducing neuronal adaptations and improving muscular output $[46,47]$. Hence, we aimed to implement a balance-based exercise intervention for CIPN patients.

In sum, the present study was undertaken to i) specify the postural abnormalities associated with CIPN during spontaneous and externally perturbed stance, ii) to identify the underlying sensorimotor malfunction, and iii) to monitor the effect of a balance-based exercise intervention in a pilot approach.

\section{Methods \\ Patients}

The present pilot study provides two approaches: a cross-sectional approach to identify postural-control differences between CIPN patients and matched healthy control subjects and a one-armed longitudinal approach to evaluate the effects of a balance-based exercise intervention on CIPN-related postural deficits.

Therefore, we examined eight cancer patients with different cancer localizations and treatment status, all reporting severe neuropathy symptoms due to chemotherapy (CIPN). The chemotherapies applied entailed the neurotoxic agents bortezomib, carboplatin, cisplatin, paclitaxal, docetaxal and vincristine. None of the patients had any neuropathy symptom before the application of neurotoxic agents. CIPN was clinically and electrophysiologically confirmed in all patients. Moreover, we assessed patients' subjective CIPN symptoms via the neurotoxicity subscale (NtxS) of FACT\&GOG (Functional Assessment of Cancer Therapy/Gynaecology Oncology Group) scored from 0 to $44(0=$ severe symptoms; $44=$ no symptoms $) ;$ Table 1 summarizes our patients' clinical information.

We excluded patients with other possible sources of neuropathy (eg hereditary, diabetes- or alcohol-induced) and patients suffering from additional deficits that might interact with their postural control such as a relevant reduction of muscular strength or certain comorbidities (eg osteolysis, severe vertebral degeneration, vestibular deficits). Specifically, all patients underwent detailed vestibular testing using a rotating chair. In addition, patients performed an incremental stress electrocardiogram on a stationary 


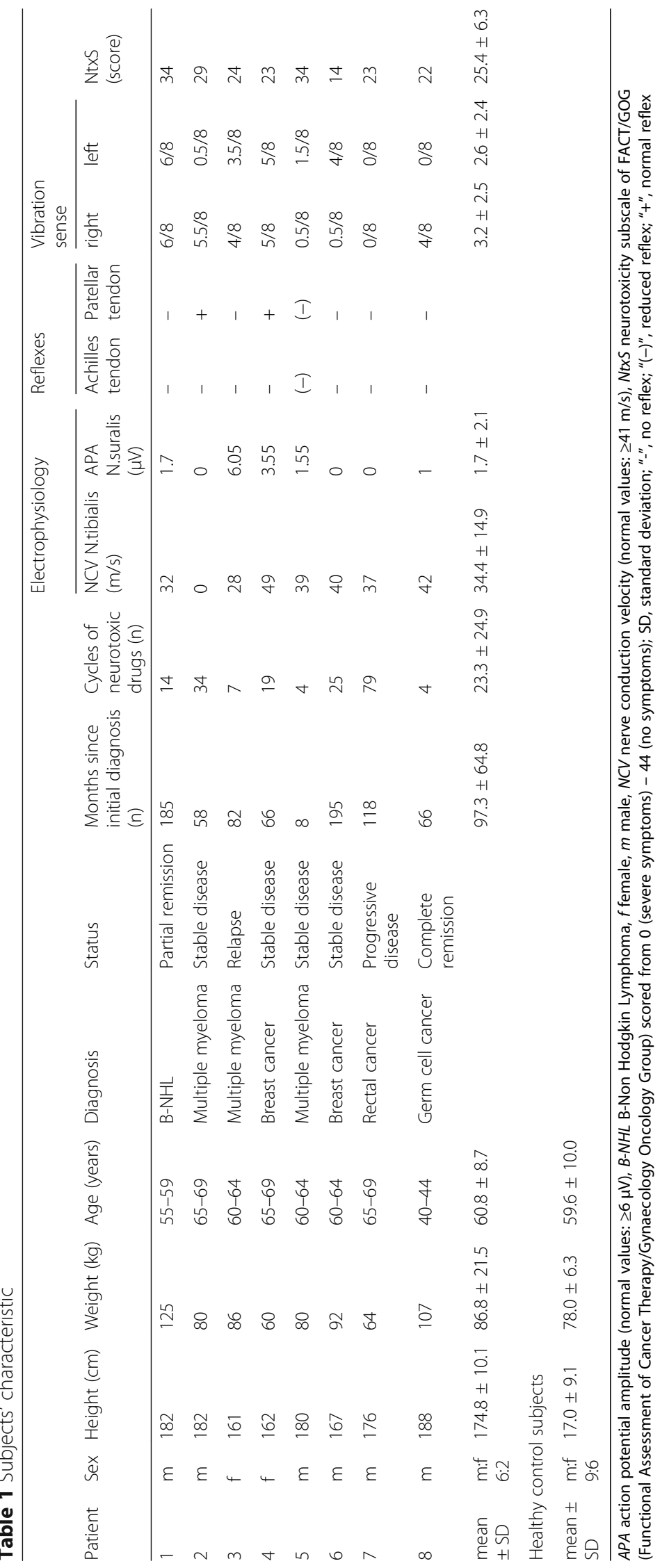


bicycle in the Institute for Exercise- and Occupational Medicine, Medical Center - University of Freiburg in order to exclude cardiovascular risks during exercise and to determine the lactate threshold for exercise control.

The control group for the postural control experiments consisted of 15 healthy subjects matched to patients' age, weight and height. We assigned two matches to each patient (except for one patient with a relative heavy body weight) to ensure a more reliable representation of the postural behavior of healthy subjects.

Patients underwent assessments of posture control twice (before and after 12 weeks of a supervised exercise intervention) while healthy control subjects underwent the assessment only once.

Patients' recruitment and data collection took place in the Clinic of Internal Medicine I and posture analyses and clinical assessments took place in the Department of Neurology and Clinical Neurophysiology, Medical Center - University of Freiburg.

The study was approved by the Ethics Commission of University of Freiburg. All subjects provided written informed consent to the experimental procedure in accordance with the Declaration of Helsinki.

\section{Intervention}

The one-on-one training sessions took place in the division of Sports Oncology in the Clinic of Internal Medicine I, twice per week over 12 weeks. The intervention protocol included a cardiovascular warm-up of up to 20 min on a stationary bicycle with an intensity of $75-80 \%$ of maximum heart rate, followed by the balance-based exercises for $30 \mathrm{~min}$ and muscular endurance training for the main muscle groups. The main focus was on the balance part of the training. Balance training prescription included a progressive increase over the intervention period in the exercise amount and difficulty. Depending on the individual performance level, that could vary during the interventions period, patient performed three (beginners) to eight exercises (more advanced) with three repetitions each à 20-30s (a 20-s rest between the repetitions and a 2-min rest between the different exercises to avoid fatigue). Moreover, exercise difficulty was also adapted to patients' performance level and successively increased by reducing the support surface (eg bipedal- to mono-pedal stance) and visual input (eyes closed), adding motor/cognitive tasks (eg moving arms or counting backwards) and inducing instability (throwing a ball or being perturbed by the sports therapist) to stimulate the sensorimotor system adequately [46, 48]. We documented vital parameters, training progress, and reasons for missed sessions.

\section{Procedure and data analysis}

For evaluating postural control, spontaneous sway and perturbed stance were measured with a custom-built motion platform $[49,50]$ under two visual conditions, with eyes open and with eyes closed. Each trial lasted 1 minute. The participants were told to stand upright on the platform in comfortable shoes. Stance width was predetermined within a marked area. For safety reasons, participants had to hold two ropes hanging from the ceiling in a crossed-arms position so that they could not perceive a somatosensory spatial orientation signal (Fig. 1a).

Data analysis was conducted off-line with custommade software programmed in $\mathrm{MATLAB}^{\circ}$ (The MathWorks Inc., Natick, MA, USA).

Spontaneous sway was measured on the non-moving platform. The center of pressure $(C O P)$ sway path was detected with a force transducing platform (Fig. 1b-d, Kistler platform type 9286, Winterthur, Switzerland). From the COP excursions over time in anterior-posterior and medio-lateral sway directions, we calculated the root mean square $(R M S)$ around the mean COP position. After differentiating the time series, we calculated mean velocity $(M V)$. In addition, center frequency $(C F)$ was extracted from the power spectrum $[51,52]$.

Perturbed stance was measured on the moving platform to differentiate sensory contributions in reaction to external disturbances. We analyzed rotational tilts in the sagittal plane with the tilt axis passing through the participant's ankle joints. Platform rotations were designed as pseudorandom stimuli (PRTS, pseudorandom ternary sequence, see Fig. 1e) [53]. This stimulus has a wide spectral bandwidth with the velocity waveform having spectral and statistical properties approximating a white noise stimulus [53]. As such, this stimulus appeared to be unpredictable to the test subject. We applied two peak angular displacements (stimulus amplitude: $0.5^{\circ}$ and $1^{\circ}$ peak-to-peak) and analyzed at eleven stimulus frequencies $(0.05,0.15,0.3,0.4,0.55,0.7,0.9,1.1,1.35,1.75$ and $2.2 \mathrm{~Hz}$ ).

Angular excursions of the lower (hip-to-ankle: hip movement) and upper (shoulder-to-hip: shoulder movement) body segments and the platform in space were measured using an optoelectronic motion-measuring device with markers attached to shoulder and hip (Optotrak 3020, Waterloo, Canada). Each marker consisted of three light-emitting diodes (LED) fixed to a rigid triangle. The triangles were fixed to the participant's hips and shoulders and to a rigid bar on the platform (Fig. 1a). 3-D LED positions of the triangles were used to calculate marker positions (Fig. 1f, g). Optotrak ${ }^{\bullet}$ and Kistler ${ }^{\bullet}$ output signals as well as the stimulus signals were sampled at $100 \mathrm{~Hz}$ using an analogue-digital converter. We recorded all data with software programmed in LabView (National Instruments, Austin, Texas, USA).

To analyse postural reactions in relation to platform stimuli, transfer functions from stimulus-response data 


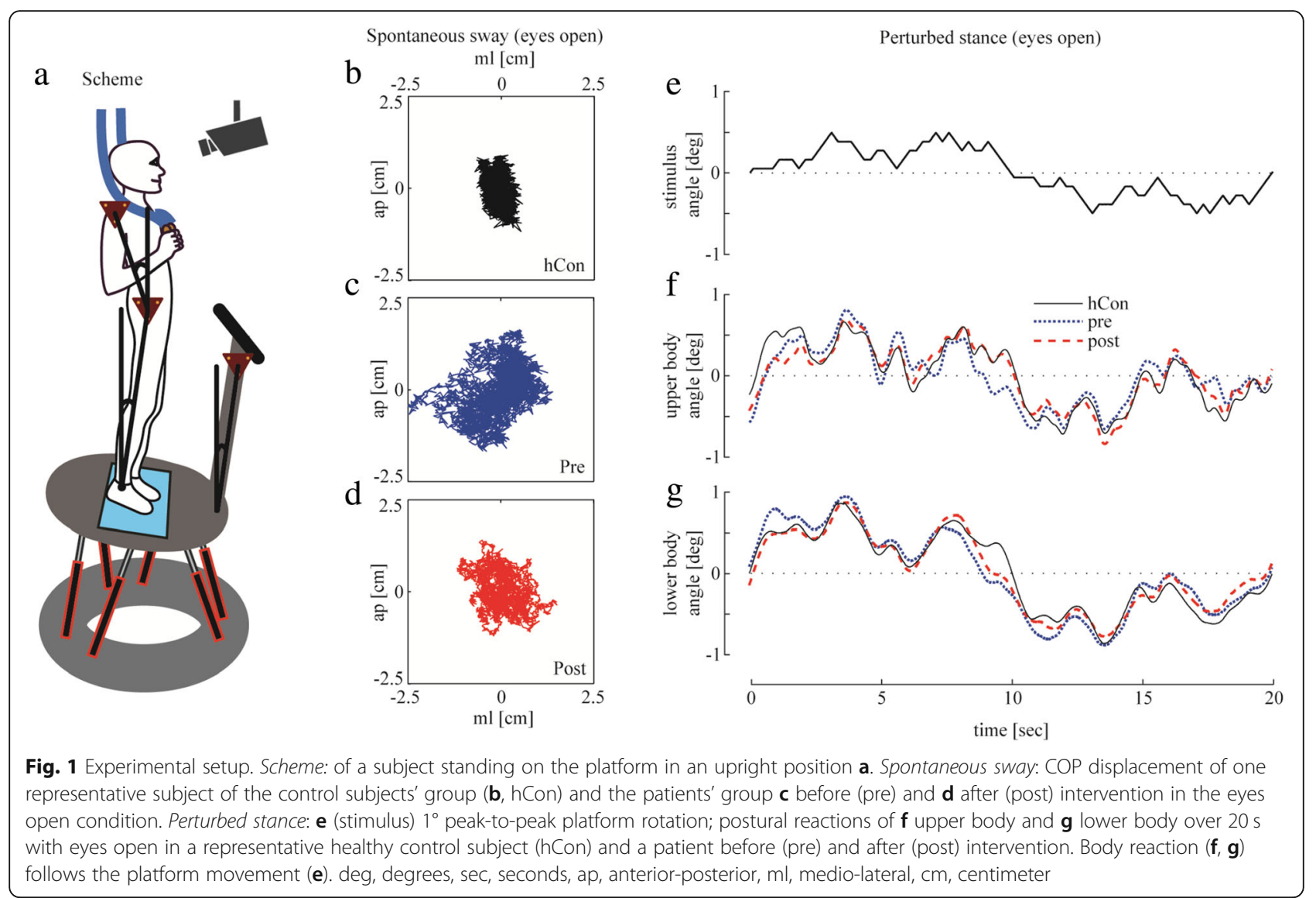

were calculated via a discrete Fourier transform. Fourier coefficients of stimulus and response time series are used to determine GAIN and PHASE with respect to stimulus frequencies. GAIN represents the size of the postural reaction as a function of stimulus size (platform angle), while PHASE is related to the relative timing between postural reaction and stimulus [54].

Furthermore, we calculated COHERENCE, a measure of reproducibility of the response. Technically, COHER$E N C E$ is calculated as the quotient between the cross power spectrum of stimulus and response, and the product of the individual spectra of stimulus and response [53]. Whereas a COHERENCE value of 0 indicates that there is no linear correlation between the stimulus and response, and 1 indicating a perfect linear correlation with no noise. Values less than 1 occur in practice either because there is noise in the system or there is a nonlinear relation between stimulus and response.

\section{Parameter identification}

Transfer functions served as the experimental data basis for model simulations using a specific version of an established postural control model [36, 49, 53, 55-57] with active time-delayed proportional, derivative, and integral feedback as well as passive stiffness and damping to extract basic constituents of postural control. The physical part of the model is a single inverted pendulum model with corrective torque applied at the ankle joint. The model used here includes a negative feedback loop that relates body excursion detected by visual, vestibular, and proprioceptive sensors to a corrective torque via a neural controller. The neural controller represents the relation between sensory error, ie the difference between the current and desired position on the one hand, and the strength of the motor output, ie torque, on the other hand. With the help of an automated optimization tool (fmincon, MATLAB ${ }^{\circ}$, The MathWorks Inc.), which minimized the difference between experimental and simulated GAIN and PHASE curves, we estimated the neural controller's parameters with proportional $(K p)$, derivative $(K d)$ and integral $(K i)$ contributions (PDI-controller). Neural controller gains are, in part, determined by mass and height of each subject's center of mass [53]. Because our control group presented lower masses and heights than patients, we had to correct neural controller gains for this effect. That is why we provide numbers for $(K p / m g h),(K d / m g h)$, and $(K i / m g h)$, where mgh represents the gravitational pull (mass)*(gravitational constant)*(height of center of mass). Moreover, we derived time delay $(T d)$, proprioceptive sensory weight $(W p)$, 
and biomechanical elasticity (Ppas) and damping (Dpas) of the muscles and tendons. We fitted model simulations to experimental transfer functions under different stimulus amplitudes and visual conditions.

\section{Statistics}

Statistical analyses were performed using Microsoft Excel, JMP ${ }^{\circ}$ and Statview (SAS Institute Inc., Cary, NC, USA). We applied parametric methods after testing the normal distribution and homogeneity of variances with the Kolmogorov-Smirnov test. Due to the expected dependency between experimental conditions and outcome measures, statistical significance was tested by an analysis of variance (ANOVA) for the comparison of healthy subjects and patients. Visual condition, sway direction, and body segment (hip, shoulder) were the withinsubjects' factors for spontaneous sway. For perturbed stance, we applied visual condition, stimulus amplitude, stimulus frequency, and body segment (hip, shoulder) as within-subjects' factors. For the analysis of the balance based exercise intervention effect on patients, we used a multivariate analysis of variance (MANOVA) with a time as the repeated measure variable, in addition. The level of statistical significance was set at $p=0.05$.

\section{Results}

No adverse events were observed during the study period. The intervention compliance in terms of number of sessions performed by the patients was $70.1 \%$, mainly due to the underlying disease.

\section{Spontaneous sway}

The patient group before intervention displayed a significantly larger COP RMS than control subjects (Fig. 2a-b and Table 2). Group designation significantly interacted with sway direction, ie the difference between control subjects and CIPN patients is larger in anteriorposterior direction. Moreover, group designation significantly interacted with visual condition, due to the large RMS in patients with eyes closed. After intervention, RMS did not change significantly.

As with RMS, the pre-intervention MV of the patient group was significantly larger than in control subjects (Figs. 2c-d, Table 2). The group designation significantly interacted with visual condition (see Table 2): MV values did not differ between groups in the eyes-open condition, whereas the patients' MV was significantly larger in the eyes-closed condition. After intervention, MV did not change.

CF did not differ significantly between patients and control subjects (Figs. 2e-f, Table 2). After intervention, patients displayed no effects on CF.

\section{Perturbed stance}

The transfer function between platform tilt and body angular displacement is characterized by GAIN and PHASE behavior.

The disturbance-induced body sway, ie GAIN was significantly smaller in patients before intervention (1.57) compared to control subjects $(1.87 ; \mathrm{F}=62.3 ; p<0.0001$; Fig. 3a). After intervention, patients' GAIN increased significantly (1.63; $\mathrm{F}=18.0 ; p<0.0001$; Fig. 3a, Fig. 4a-d). Furthermore, group designation interacted significantly with stimulus frequency $(\mathrm{F}=3.70 ; p<0.0001)$, due to a distortion of the transfer function (Fig. 3a, Fig. 4a-d). Moreover, control subjects' GAIN is larger with closed eyes than open eyes whereas patients' GAIN was almost similar independent of the visual condition: group designation significantly interacted with visual condition (eyes open: control subjects 1.58; patients before intervention 1.46; after intervention 1.51; eyes closed: control subjects 2.15; patients before intervention 1.67; after intervention 1.74; visual condition: $\mathrm{F}=25.6 ; p<0.0001$, Fig. 3d, Fig. 4a-d). The difference between shoulder and hip sway as a function of platform tilts was greater in control subjects than in patients (Fig. 3e, Fig. 4a-d): with a significant interaction between group designation and body segment $(\mathrm{F}=2.85 ; p=0.022)$. Group designation and stimulus amplitude did not interact significantly as the effect of stimulus amplitude (non-linearity) on GAIN did not differ between groups.

Concerning PHASE behavior, patients' PHASE lag before intervention was significantly less pronounced than the control group's (control subjects -118.3 , negative value patients; -107.6, negative value, $\mathrm{F}=10.3 ; p<0.0001$; Fig. $3 \mathrm{~b}$ ). After intervention, PHASE changed significantly $(-121.3$, negative value; $\mathrm{F}=15.4 ; p<0.0001$; Fig. $3 \mathrm{~b}$ ) and fell in the range of the control subjects' values (-118.3, negative value; Fig. 3b). Group designation significantly interacted with visual condition ( $\mathrm{F}=4.45, p=0.01$, Fig. $3 \mathrm{f})$ : patients with open eyes displayed a PHASE advance of 20 degrees with respect to control subjects, whereas there was no significant PHASE difference between patients and control subjects with eyes closed. Furthermore, group designation significantly interacted with body segment $(\mathrm{F}=13.1, p<0.0001$, Fig. 3g): the difference between shoulder and hip PHASE was larger in control subjects than in patients. Moreover, group designation significantly interacted with stimulus amplitude $(\mathrm{F}=9.89, p<0.0001)$ as there was a pronounced phase difference with small stimulus amplitudes. Group designation and stimulus frequency did not interact significantly $(\mathrm{F}=0.41 ; p=0.99)$ : the PHASE effects were distributed equally across all frequencies.

COHERENCE as a measure for the reproducibility of the response was smaller in patients before intervention (0.43) compared to control subjects $(0.50 ; \mathrm{F}=103 ; p<$ 0.0001; Fig. 3c). After intervention, COHERENCE did not change (0.45). However, COHERENCE significantly 

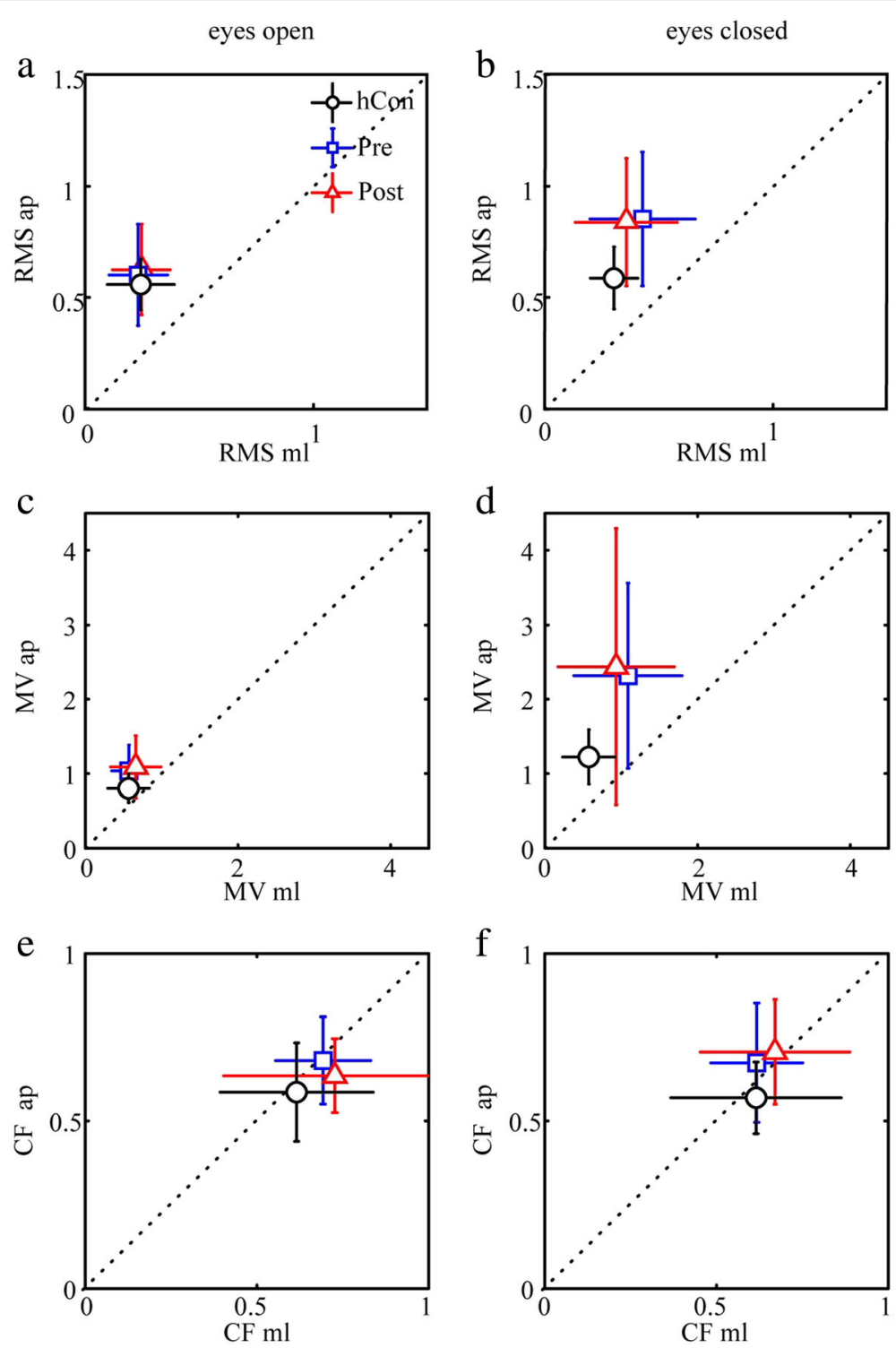

Fig. 2 Spontaneous sway. Mean and standard deviation of $\mathbf{a}$, b root mean square (RMS), $\mathbf{c}, \mathbf{d}$ mean velocity (MV) and $\mathbf{e}$, $\mathbf{f}$ center frequency (CF) of COP sway in anterior-posterior (ap) and medio-lateral ( $\mathrm{ml}$ ) direction each for the eyes-open and eyes-closed condition in healthy control subjects (hCon), patients before (pre) and after (post) intervention

varied with stimulus amplitude, frequency, visual condition, and body segment, similarly in both groups.

\section{Model-based parameter identification}

The following results are derived from the model-based parameter identification procedure [36, 49, 53, 55-57], and present the relevant parameter differences between patients and control subjects.

There was no significant effect for the integral part of the neural controller (Ki), as well as for the proportional $(\mathrm{Kp})$ and derivative part $(\mathrm{Kd})$ of the neural controller.

The sensory weighting factor Wp differed significantly between patients and control subjects $(\mathrm{F}=9.89, p=0.0001$;
Fig. 5b). Whereas patients rely with an average factor of 0.53 on proprioceptive cues and hence 0.47 on spatial cues, control subjects rely with a factor of 0.67 on proprioceptive and 0.33 on spatial cues. Group designation did not interact with visual condition or stimulus amplitude. After intervention, there was a small but significant change towards control subjects (Wp 0.56, $\mathrm{F}=9.13, p=0.006$ ).

The time delay between stimulus and response $(\mathrm{Td})$ did not differ significantly between patients and control subjects $(\mathrm{F}=1.10 ; p=0.34$; Fig. $5 \mathrm{c})$. After intervention, Td was not affected.

Parameters related to passive muscle and tendon behavior (Ppas and Dpas) did not differ significantly between 
Table 2 Spontaneous sway measures (RMS, MV, CF) with group effects, and interactions between groups and visual conditions / sway directions

\begin{tabular}{|c|c|c|c|c|c|c|}
\hline & & Healthy control subjects & Patients pre-intervention & Patients post-intervention & F-value & $p$-value \\
\hline RMS (cm) & & $0.46 \pm 0.18$ & $0.56 \pm 0.27$ & $0.53 \pm 0.27$ & 10.926 & $<0.0001$ \\
\hline visual condition & $\begin{array}{l}\text { eyes open } \\
\text { eyes closed }\end{array}$ & $\begin{array}{l}0.45 \pm 0.18 \\
0.56 \pm 0.19\end{array}$ & $\begin{array}{l}0.49 \pm 0.23 \\
0.63 \pm 0.30\end{array}$ & $\begin{array}{l}0.48 \pm 0.22 \\
0.59 \pm 0.31\end{array}$ & 5.06 & 0.007 \\
\hline sway direction & $\begin{array}{l}\text { anterior-posterior } \\
\text { medio-lateral }\end{array}$ & $\begin{array}{l}0.52 \pm 0.16 \\
0.35 \pm 0.17\end{array}$ & $\begin{array}{l}0.67 \pm 0.28 \\
0.41 \pm 0.19\end{array}$ & $\begin{array}{l}0.67 \pm 0.25 \\
0.35 \pm 0.18\end{array}$ & 4.06 & 0.018 \\
\hline $\mathrm{MV}(\mathrm{cm} / \mathrm{s})$ & & $0.35 \pm 0.14$ & $0.69 \pm 0.57$ & $0.69 \pm 0.56$ & 7.80 & 0.0005 \\
\hline visual condition & $\begin{array}{l}\text { eyes open } \\
\text { eyes closed }\end{array}$ & $\begin{array}{l}0.32 \pm 0.13 \\
0.39 \pm 0.14\end{array}$ & $\begin{array}{l}0.45 \pm 0.31 \\
0.92 \pm 0.84\end{array}$ & $\begin{array}{l}0.50 \pm 0.35 \\
0.88 \pm 0.81\end{array}$ & 8.00 & 0.0004 \\
\hline sway direction & $\begin{array}{l}\text { anterior-posterior } \\
\text { medio-lateral }\end{array}$ & $\begin{array}{l}0.41 \pm 0.13 \\
0.27 \pm 0.12\end{array}$ & $\begin{array}{l}0.82 \pm 0.78 \\
0.51 \pm 0.44\end{array}$ & $\begin{array}{l}0.84 \pm 0.73 \\
0.49 \pm 0.44\end{array}$ & & n.s. \\
\hline $\mathrm{CF}(\mathrm{Hz})$ & & $0.37 \pm 0.11$ & $0.46 \pm 0.18$ & $0.46 \pm 0.21$ & & n.s. \\
\hline
\end{tabular}

RMS root means square, $M V$ mean velocity, $C F$ center frequency, $n . s$ not significant

F- and $p$-values refer to the comparison between the three groups and subordinated, to the post-hoc analysis of the interaction with visual condition and sway direction, respectively

groups. After intervention, these parameters were not affected.

Figure 5d shows the modified postural-control model.

\section{Discussion}

As postural instability is a momentous symptom of CIPN [9-16], the first aim of this study was to assess the specific set of postural control deficits associated with CIPN compared to healthy subjects. Furthermore, since CIPN treatment options are very limited so far [3] and hints in the recent literature indicate that CIPN patients might benefit from exercising $[39,41,58]$, we evaluated a balance-based exercise intervention aiming to treat patients' functional impairments due to CIPN. While former studies mostly investigated spontaneous sway measures (displacement-, velocity-, and frequencyrelated measures), we aimed to describe CIPN patients' sensorimotor behavior in much greater detail. Therefore, we additionally analyzed patients' stance behavior as reaction to an external perturbation (following a pseudorandom stimuli) by generating transfer functions between body behavior and stimuli.

\section{Spontaneous sway}

Concerning spontaneous sway, we found greater postural sway in CIPN patients similarly to previous CIPN studies [10, 12, 14, 39]. Additionally, our findings correspond to that of other types of neuropathy. For example, many working groups [59-63] report increased RMS and MV in patients with diabetic-induced neuropathy. In our study, RMS and MV were significantly larger in CIPN patients than in healthy subjects. Moreover, we observed a specific preponderance of deficits in anteriorposterior direction [60] and a more pronounced postural sway with closed rather than open eyes [60, 61]. Generally, closing their eyes causes subjects to use vestibular and proprioceptive cues for controlling balance. As proprioceptive information are often deficient in neuropathy patients [26], it seems reasonable to assume that patients may prefer vestibular over proprioceptive cues. However, it is well known that the vestibular signal carries a larger amount of noise than the proprioceptive signal [64] leading to less accurate posture control. We speculate at this point that the main source for the larger RMS and MV is related to a sensory shift towards vestibular cues (see below sensory weighting, and [65]). If that is true, we might be able to recover this finding when dissociating proprioceptive from vestibular frames of orientation using platform tilts.

\section{Perturbed stance}

Whereas proprioceptive cues may drag the body along platform movements, quantified by a relative larger GAIN as transfer function between body excursions and platform tilts, vestibular cues would stabilize the body in space, quantified by a relative smaller GAIN. In fact, CIPN patients presented smaller GAIN values since their reaction to platform tilts were less pronounced than that of control subjects. Thus, they might rather use space coordinates than platform movements for posture control. Furthermore, GAIN was significantly affected by visual condition and body segment. The larger GAIN difference between CIPN patients and control subjects in the eyesclosed compared to the eyes-open condition suggests that under-usage of proprioception is dominant when there are less additional orienting cues. Moreover, the finding of relatively small GAINs in CIPN patients' lower compared to their upper body segment point to a slightly different intersegmental strategy [29]. Our PHASE finding, that the difference between shoulder and hip PHASE was larger in control subjects than in patients, also points to a different intersegmental behavior in terms of upper with respect to 

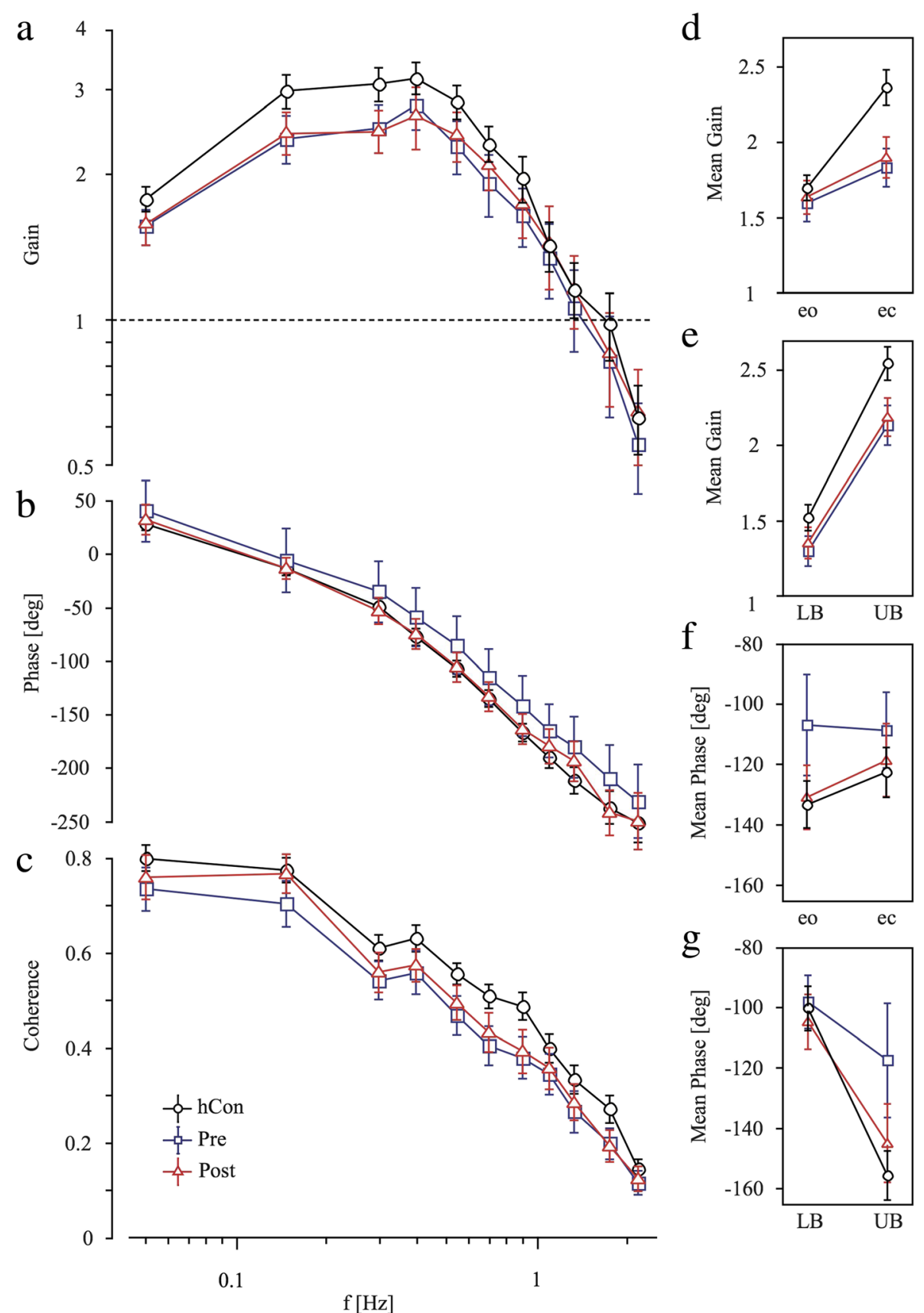

Fig. 3 Transfer functions. Mean and standard deviation of $\mathbf{a}$ Gain, $\mathbf{b}$ Phase and $\mathbf{c}$ Coherence behavior as a function of frequency ( $f$ ) and $\mathbf{d}$, e mean Gain and $\mathbf{f}, \mathbf{g}$ mean Phase behavior for lower body (LB) and upper body (UB) and for eyes-open (eo) and eyes-closed (ec) condition in healthy control subjects (hCon), patients before (pre) and after (post) intervention

lower body angular displacements [26, 28, 29]. We assume that patients proactively orientate themselves, especially their upper body, more towards space coordinates. This also indicates an especially low use of proprioception according to our GAIN results. Moreover, we speculate that CIPN patients proactively assume a safety strategy that may follow an enhanced muscle co-contraction [25], leading to smaller body excursions. However, greater co-contraction limits one's ability to precisely control posture $[66,67]$.

\section{Model-based parameter identification}

To address the transfer-function abnormalities in CIPN patients, we fitted the subjects' data via a simple feedback system [36, 56, 64, 68]. Using the model-based parameter estimation, we identified and quantified the 

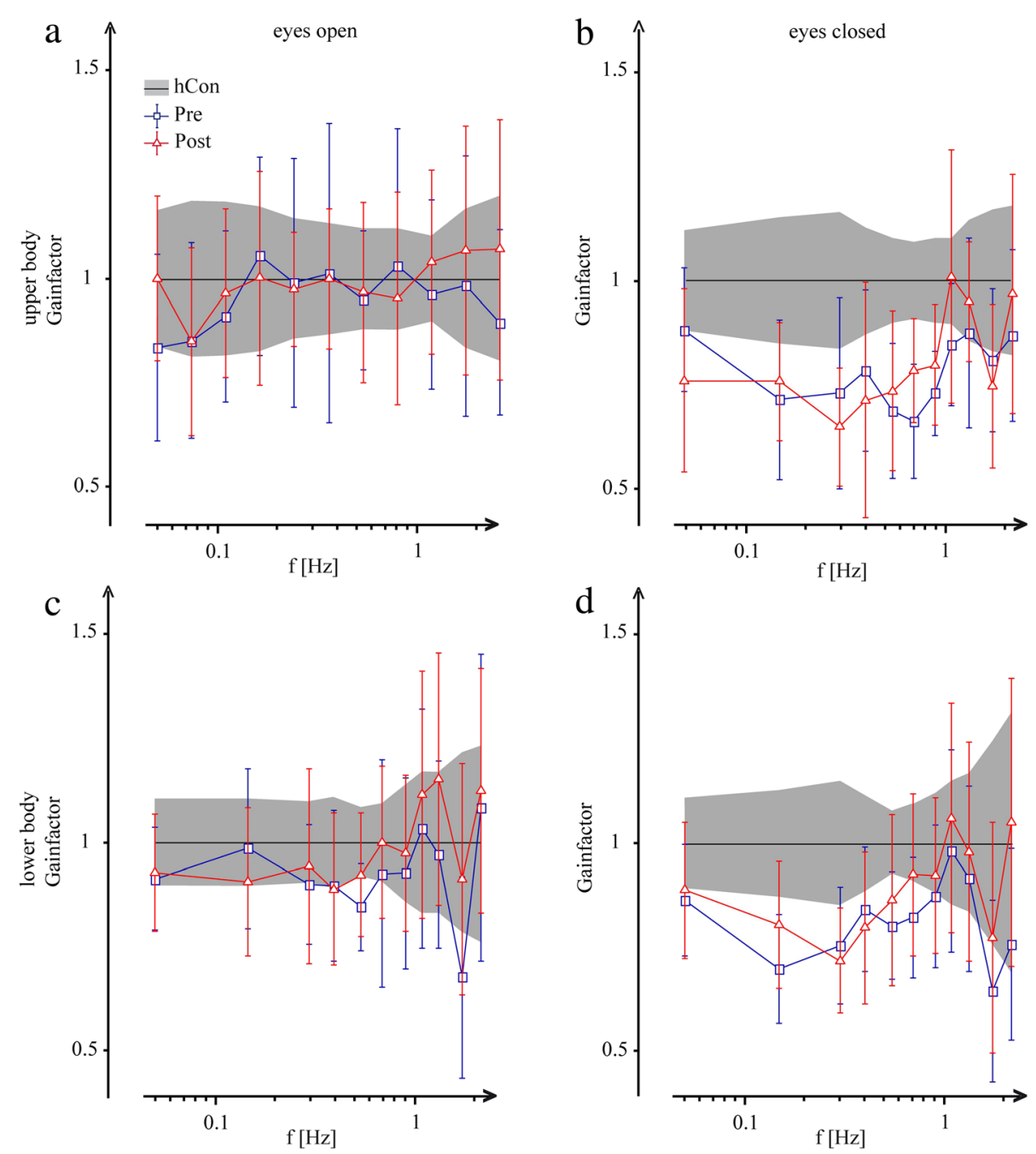

Fig. 4 Gainfactor. Mean and standard deviation of $\mathbf{a}$, $\mathbf{b}$ upper body and $\mathbf{c}$, $\mathbf{d}$ lower body GAINFACTOR behavior of patients before (pre) and after (post) intervention as a function of frequency ( $f$ ). GAINFACTOR represents patients' GAIN values normalized to values of healthy control subjects ( $\mathrm{hCon}$ ) in the eyes-open and eyes-closed condition

CIPN patients' diminished use of proprioceptive cues: The sensory-weighting factor for proprioception (Wp) is significantly smaller in CIPN patients than in control subjects. However, patients did not present a different error correction gain $(\mathrm{Kp}$ and $\mathrm{Kd}$ ) of the feedback loop. Furthermore, parameters related to passive muscle and tendon behavior (passive stiffness and damping, Kpas and Bpas) did also not differ between CIPN patients and control subjects. This seems to be in line with the notion that differences in postural control between CIPN patients and healthy subjects mainly rely on active postural control differences related to the different use of sensory cues, whereas passive muscle and tendon characteristics are not significantly affected by CIPN. Moreover, this finding might indicate that muscles and tendons were not affected supporting CIPN's primarily sensory characteristic.

\section{Intervention effects}

Our patients performed more than two third of the prescribed exercise sessions. Considering the high variety of our patient group with different diagnosis and disease severities, we assume a reasonable coherence rate that lies in the range of other interventional studies with cancer patients in general [69] or CIPN patients specifically [41].

How does the exercise intervention influence CIPN patients' postural behavior? Interestingly, we observed that CIPN patients' main abnormality (down-weighted proprioception) was modified by the exercise intervention. CIPN patients' GAIN and PHASE values reached those of healthy subjects due to the proprioceptive up-weighting mentioned above. Interestingly, the effect of up-weighting proprioception is the only significant model-based parameter modification after intervention. Why would CIPN patients profit from up-weighting proprioception while 


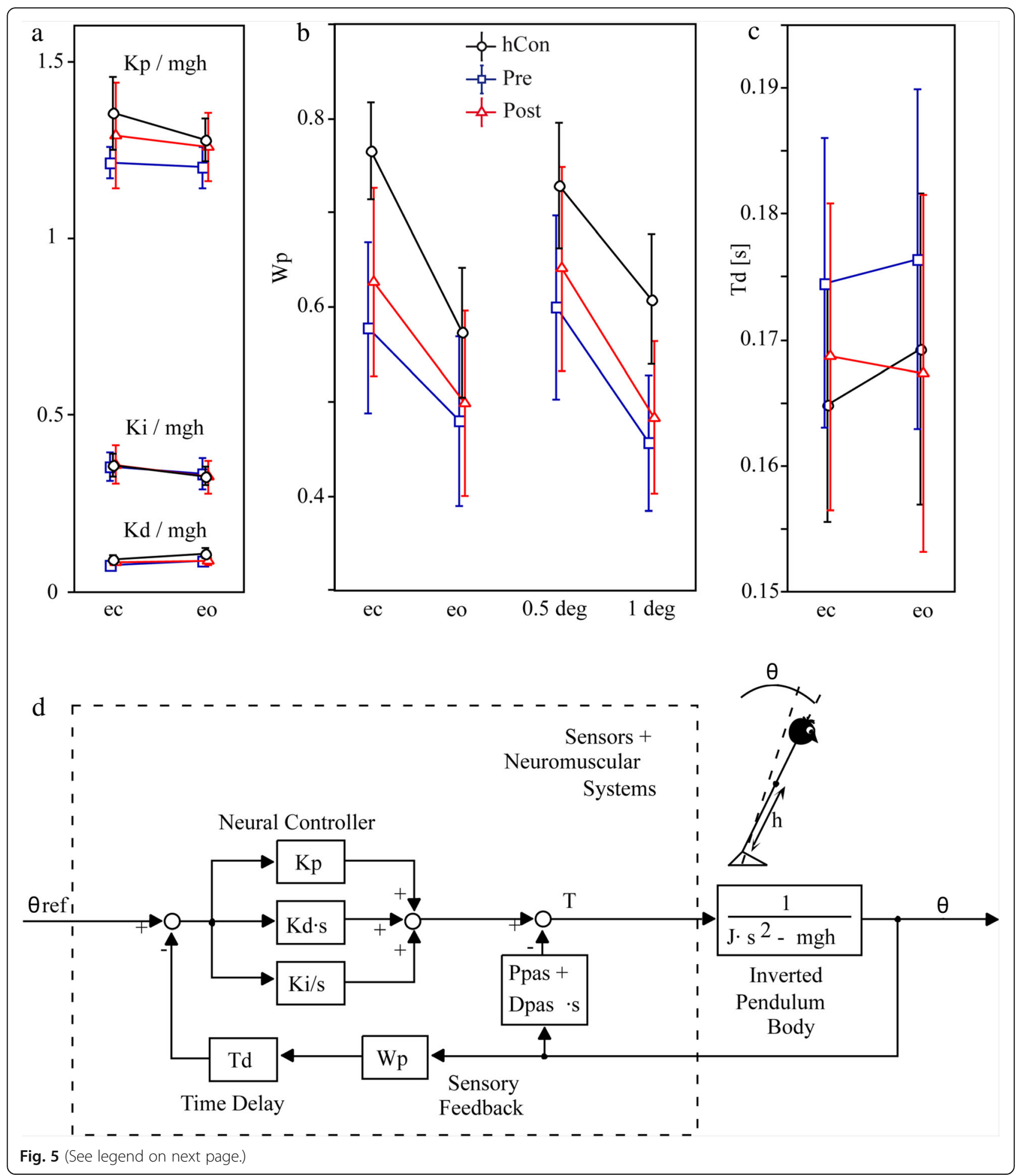


(See figure on previous page.)

Fig. 5 Model parameters. Mean and standard deviation of a the neural controller with the proportional $\left(\mathrm{Kp} / \mathrm{mgh}\right.$ in $\left.1 /{ }^{\circ}\right)$, derivative $(\mathrm{Kd} / \mathrm{mgh}$ in $\mathrm{s} /$ ${ }^{\circ}$ ) and integral (Ki/mgh in $1 / \mathrm{s}^{* 0}$ ) contribution corrected for subjects' masses and heights, of $\mathbf{b}$ the proprioceptive sensory weight (Wp in $\%{ }^{\circ}$ ) and $\mathbf{c}$ the lumped time delay (Td in seconds) for healthy control subjects (hCon), patients before (pre) and after (post) intervention, each shown in the eyes-open (eo) and eyes-closed (ec) condition and for $\mathbf{b}$ Wp in 0.5 and 1degree (deg) platform rotation. $\mathbf{d}$ shows the modified postural-control model used to identify abnormal postural control parameters in CIPN patients via an optimization procedure where differences between experimental data and model simulations were minimized: The model consists of a body represented by an inverted pendulum with the mass concentrated at the center of mass of the body and the sensors and neuromuscular systems including a Neural Controller. $\theta$, body sway angle; $h$, height of the center of mass above the ankle joints; $\theta$ ref., external stimulus; Kp, proportional gain (stiffness factor), Kd, derivative gain (damping factor), Ki, integral gain of the Neural Controller; Ppas, passive stiffness factor; Dpas, passive damping factor; Wp, proprioceptive sensory weight; $\mathrm{Td}$, feedback time delay; $\mathrm{T}$, control torque; J, moment of inertia of the body; mgh, body mass* gravitational constant*height of the center of mass from the ankle joint; s, Laplace transform variable

suffering from a supposed proprioceptive deficit? Our clinical assessments did not suffice to conclusively specify neural lesions or identify CIPN's nerve fiber contribution occurring in our patients. However, all patients suffered from strong paresthesia and reported significant balance problems confirmed by our spontaneous sway experiments. CIPN sensory symptoms are described to refer to 'terminal arbor degeneration' and the preferential damage of myelinated primary afferent sensory nerve fibers $[2,70]$. Therefore, we speculate that patients' peripheral information and subsequent stimulus conduction are altered, but not completely dysfunctional. The down-weighting of proprioceptive cues could be interpreted as an excessive compensatory mechanism, which lets CIPN patients pre-intervention remain in a suboptimal state. The exercise intervention may thus partially correct this excess and may stimulate the use of less damaged pathways. As a side effect of proprioceptive upweighting, the intervention may trigger downweighting of vestibular cues, thereby reducing vestibular noise. As a consequence, postural-control behavior might be more accurate in terms of less variability.

Conclusively, we maintain that up-weighting proprioception and thereby down-weighting vestibular information towards the behavior of healthy subjects represents a clear benefit for CIPN patients. The proprioceptive cue is considered to be more accurate than vestibular cues for postural stability [64]. Regarding time delay, patients after intervention tended to improve their reaction time between stimulus and response. We also identified a tendency of spontaneous sway RMS values to be smaller after intervention, being in line with postural sway findings after balance training in a study of Schwenk et al. [39]. Although, our intervention effects are small, we are convinced that CIPN patients benefit from exercising in terms of improved posture behavior that substantially contributes to patients' functional status. Functional performance is an acknowledged prognosis factor for cancer survivor [71] why we strongly propose to verify our intervention results in a greater randomized controlled trial. Our findings are limited by the small sample size and the lack of patient control group. Furthermore, expanding neurophysiological assessments would provide insights in adaptive processes unexamined in this study.

\section{Conclusion}

We believe that our new approach contributed to a deeper understanding of CIPN patients' postural instability. Proprioceptive down-weighting might represent the main postural deficit in CIPN. Our exercise intervention targeted specifically this abnormality presumably by primarily correcting the overactive compensation, which led to a significant improvement in postural stability. We believe that a balance-based exercise intervention is a promising strategy to manage functional impairments due to CIPN and that it should therefore be routinely integrated within the treatment regimens of patients receiving neurotoxic agents.

\section{Abbreviations \\ CF: Center frequency; CIPN: Chemotherapy-induced peripheral neuropathy; COP: Center of pressure; Dpas: Passive damping; Kd: Derivative contribution of the neural controller; Ki: Integral contribution of the neural controller; Kp: Proportional contribution of the neural controller; MV: Mean velocity; NtxS: Neurotoxicity subscale of FACT\&GOG; Ppas: Passive stiffness; RMS: Root means square; Td: Time delay; Wp: Proprioceptive sensory weight}

\section{Acknowledgements}

We acknowledge the cooperation and training implementation of sports- and physiotherapists of the Sports Oncology of Department of Medicine I, Medical Center - University of Freiburg and we thank the patients and control individuals for their collaboration. The article processing charge was funded by the German Research Foundation (DFG) and the Albert Ludwigs University Freiburg, Germany, in the funding program Open Access Publishing.

\section{Authors' contributions}

SK, AW and CM contributed to the conception of the work, to data acquisition, analysis and interpretation, wrote the first draft and revised it. IW, DD, and JL contributed to data acquisition and analysis and revised the work critically. AG and $\mathrm{HB}$ contributed to the design of the work and revised it critically. All authors approved the final version of this article and agree to be accountable for all aspects of the work in ensuring that questions related to the accuracy or integrity of any part of the work are appropriately investigated and resolved.

\section{Funding}

C.M. was partially funded by the European Union grants FP7 H2020-SC1-2017CNECT-1 (HOLOBALANCE: Project ID 769574; HOLOgrams for personalised virtual coaching and motivation in an ageing population with BALANCE disorders)

\section{Availability of data and materials}

The dataset supporting the conclusions of this article is included within this article. The data that support the findings of this study are available from the corresponding author upon reasonable request. 


\section{Ethics approval and consent to participate}

This study was approved by the Ethics Committee of the University of Freiburg (478/11). All procedures performed in studies involving human participants were in accordance with the ethical standards of the institutional and/or national research committee and with the 1964 Helsinki declaration and its later amendments or comparable ethical standards. Written informed consent was obtained from all individual participants included in the study.

\section{Consent for publication}

Not applicable.

\section{Competing interests}

The authors declare that the research was conducted in the absence of any commercial or financial relationships that could be construed as a potential conflict of interest.

\section{Author details}

${ }^{1}$ Department of Neurology and Neuroscience, Faculty of Medicine, Medical Center, University of Freiburg, Freiburg, Germany. ${ }^{2}$ Institute for Exercise- and Occupational Medicine, Faculty of Medicine, Medical Center, University of Freiburg, Freiburg, Germany. ${ }^{3}$ Department of Sports and Sport Science, University of Freiburg, Freiburg, Germany.

\section{Received: 16 September 2019 Accepted: 26 December 2019} Published online: 16 January 2020

\section{References}

1. Argyriou AA, Kyritsis AP, Makatsoris T, Kalofonos HP. Chemotherapy-induced peripheral neuropathy in adults: a comprehensive update of the literature. Cancer Manag Res. 2014;6:135-47.

2. Han Y, Smith MT. Pathobiology of cancer chemotherapy-induced peripheral neuropathy (CIPN). Front Pharmacol. 2013;4:156.

3. Hershman DL, Lacchetti C, Dworkin RH, Smith EML, Bleeker J, Cavaletti G, et al. Prevention and Management of Chemotherapy-Induced Peripheral Neuropathy in survivors of adult cancers: American Society of Clinical Oncology clinical practice guideline. J Clin Oncol. 2014;32(18):1941-67.

4. Gutiérrez-Gutiérrez G, Sereno M, Miralles A, Casado-Sáenz E, GutiérrezRivas E. Chemotherapy-induced peripheral neuropathy: clinical features diagnosis, prevention and treatment strategies. Clin Transl Oncol. 2010; 12(2):81-91.

5. Stubblefield MD, Burstein HJ, Burton AW, Custodio CM, Deng GE, Ho M, et al. NCCN task force report: management of neuropathy in cancer. J Natl Compr Cancer Netw JNCCN. 2009;7(Suppl 5):S1-26 quiz S27-28.

6. Windebank AJ, Grisold W. Chemotherapy-induced neuropathy. J Peripher Nerv Syst. 2008;13(1):27-46.

7. Pike CT, Birnbaum HG, Muehlenbein CE, Pohl GM, Natale RB. Healthcare costs and workloss burden of patients with chemotherapy-associated peripheral neuropathy in breast, ovarian, head and neck, and nonsmall cell lung cancer. Chemother Res Pract. 2012;2012:913848.

8. Asbury A, Thomas P. Peripheral Nerve Disorders 2. Oxford: ButterworthHeinemann; 1995. p. 336

9. Tofthagen C, Overcash J, Kip K. Falls in persons with chemotherapy-induced peripheral neuropathy. Support Care Cancer. 2012;20(3):583-9.

10. Wampler MA, Topp KS, Miaskowski C, Byl NN, Rugo HS, Hamel K. Quantitative and clinical description of postural instability in women with breast Cancer treated with Taxane chemotherapy. Arch Phys Med Rehabil. 2007;88(8):1002-8.

11. Winters-Stone KM, Horak F, Jacobs PG, Trubowitz P, Dieckmann NF, Stoyles $S$, et al. Falls, functioning, and disability among women with persistent symptoms of chemotherapy-induced peripheral neuropathy. J Clin Oncol. 2017;35(23):2604-12

12. Herman HK, Monfort SM, Pan XJ, Chaudhari AMW, Lustberg MB. Effect of chemotherapy-induced peripheral neuropathy on postural control in cancer survivors. J Clin Oncol. 2017;35(5_suppl):128.

13. Marshall TF, Zipp GP, Battaglia F, Moss R, Bryan S. Chemotherapy-inducedperipheral neuropathy, gait and fall risk in older adults following cancer treatment. J Cancer Res Pract. 2017:4(4):134-8.

14. Monfort SM, Pan X, Patrick R, Ramaswamy B, Wesolowski R, Naughton MJ, et al. Gait, balance, and patient-reported outcomes during taxane-based chemotherapy in early-stage breast cancer patients. Breast Cancer Res Treat. 2017;164(1):69-77.

15. Gewandter JS, Fan L, Magnuson A, Mustian K, Peppone L, Heckler C, et al. Falls and functional impairments in cancer survivors with chemotherapyinduced peripheral neuropathy (CIPN): a University of Rochester CCOP study. Support Care Cancer. 2013;21(7):2059-66.

16. Bao T, Basal C, Seluzicki C, Li SQ, Seidman AD, Mao JJ. Long-term chemotherapy-induced peripheral neuropathy among breast cancer survivors: prevalence, risk factors, and fall risk. Breast Cancer Res Treat. 2016; 159(2):327-33.

17. Kolb NA, Smith AG, Singleton JR, Beck SL, Stoddard GJ, Brown S, et al. The Association of Chemotherapy-Induced Peripheral Neuropathy Symptoms and the risk of falling. JAMA Neurol. 2016;73(7):860-6.

18. Mols F, Beijers T, Vreugdenhil G, van de Poll-Franse L. Chemotherapyinduced peripheral neuropathy and its association with quality of life: a systematic review. Support Care Cancer. 2014;22(8):2261-9.

19. Cesari M, Kritchevsky SB, Newman AB, Simonsick EM, Harris TB, Penninx BW, et al. Added value of physical performance measures in predicting adverse health-related events: results from the health, aging and body composition study. J Am Geriatr Soc. 2009;57(2):251-9.

20. Cesari M, Cerullo F, Zamboni V, Palma RD, Scambia G, Balducci L, et al. Functional status and mortality in older women with gynecological Cancer. J Gerontol A Biol Sci Med Sci. 2013;68(9):1129-33.

21. Klepin HD, Geiger AM, Tooze JA, Newman AB, Colbert LH, Bauer DC, et al. Physical performance and subsequent disability and survival in older adults with malignancy: results from the health, aging and body composition study. J Am Geriatr Soc. 2010;58(1):76-82.

22. Onder G, Penninx BWJH, Ferrucci L, Fried LP, Guralnik JM, Pahor M. Measures of physical performance and risk for progressive and catastrophic disability: results from the Women's health and aging study. J Gerontol A Biol Sci Med Sci. 2005;60(1):74-9.

23. Alekna V, Stukas R, Tamulaitytè-Morozovienè I, Šurkienè G, Tamulaitienè M. Self-reported consequences and healthcare costs of falls among elderly women. Medicina (Kaunas). 2015;51(1):57-62.

24. Alexander BH, Rivara FP, Wolf ME. The cost and frequency of hospitalization for fall-related injuries in older adults. Am J Public Health. 1992;82(7):1020-3.

25. Kneis S, Wehrle A, Freyler K, Lehmann K, Rudolphi B, Hildenbrand B, et al. Balance impairments and neuromuscular changes in breast cancer patients with chemotherapy-induced peripheral neuropathy. Clin Neurophysiol. 2016;127(2):1481-90

26. Bonnet C, Carello C, Turvey MT. Diabetes and postural stability: review and hypotheses. J Mot Behav. 2009;41:172-92.

27. Dickstein R, Peterka RJ, Horak FB. Effects of light fingertip touch on postural responses in subjects with diabetic neuropathy. J Neurol Neurosurg Psychiatry. 2003;74(5):620-6.

28. Dickstein $\mathrm{R}$, Shupert $\mathrm{CL}$, Horak FB. Fingertip touch improves postural stability in patients with peripheral neuropathy. Gait Posture. 2001;14(3):238-47.

29. Simmons RW, Richardson C, Pozos R. Postural stability of diabetic patients with and without cutaneous sensory deficit in the foot. Diabetes Res Clin Pract. 1997;36(3):153-60.

30. Horak FB, Hlavacka F. Somatosensory loss increases vestibulospinal sensitivity. J Neurophysiol. 2001;86(2):575-85.

31. Fitzpatrick R, McCloskey DI. Proprioceptive, visual and vestibular thresholds for the perception of sway during standing in humans. J Physiol. 1994; 478(Pt 1):173-86.

32. Nashner LM, Black FO, Wall C. Adaptation to altered support and visual conditions during stance: patients with vestibular deficits. J Neurosci. 1982; 2(5):536-44.

33. Peterka RJ, Benolken MS. Role of somatosensory and vestibular cues in attenuating visually induced human postural sway. Exp Brain Res. 1995; 105(1):101-10.

34. Corna S, Tarantola J, Nardone A, Giordano A, Schieppati M. Standing on a continuously moving platform: is body inertia counteracted or exploited? Exp Brain Res. 1999;124(3):331-41.

35. Schieppati M, Giordano A, Nardone A. Variability in a dynamic postural task attests ample flexibility in balance control mechanisms. Exp Brain Res. 2002; 144(2):200-10

36. Engelhart D, Pasma JH, Schouten AC, Meskers CGM, Maier AB, Mergner $T$ et al. Impaired standing balance in elderly: a new engineering method helps to unravel causes and effects. J Am Med Dir Assoc. 2014;15(3): 227.e1-6. 
37. Pasma $J H$, Engelhart $D$, Maier $A B$, Schouten $A C$, van der Kooij $H$, Meskers CGM. Changes in sensory reweighting of proprioceptive information during standing balance with age and disease. J Neurophysiol. 2015;114(6):3220-33.

38. Wiesmeier IK, Dalin D, Maurer C. Elderly use proprioception rather than visual and vestibular cues for postural motor control. Front Aging Neurosci. 2015;7:97.

39. Schwenk M, Grewal GS, Holloway D, Muchna A, Garland L, Najafi B. Interactive sensor-based balance training in older Cancer patients with chemotherapy-induced peripheral neuropathy: a randomized controlled trial. Gerontology. 2016;62(5):553-63.

40. Streckmann F, Zopf EM, Lehmann HC, May K, Rizza J, Zimmer P, et al. Exercise intervention studies in patients with peripheral neuropathy: a systematic review. Sports Med. 2014;44(9):1289-304.

41. Zimmer P, Trebing S, Timmers-Trebing U, Schenk A, Paust R, Bloch W, et al. Eight-week, multimodal exercise counteracts a progress of chemotherapyinduced peripheral neuropathy and improves balance and strength in metastasized colorectal cancer patients: a randomized controlled trial. Support Care Cancer. 2018;26(2):615-24.

42. Kneis S, Wehrle A, Müller J, Maurer C, Ihorst G, Gollhofer A, et al. It's never too late - balance and endurance training improves functional performance, quality of life, and alleviates neuropathic symptoms in cancer survivors suffering from chemotherapy-induced peripheral neuropathy: results of a randomized controlled trial. BMC Cancer. 2019;19(1):414.

43. Allet L, Armand S, de Bie RA, Golay A, Monnin D, Aminian K, et al. The gait and balance of patients with diabetes can be improved: a randomised controlled trial. Diabetologia. 2010;53(3):458-66.

44. Lee K, Lee S, Song C. Whole-body vibration training improves balance, muscle strength and glycosylated hemoglobin in elderly patients with diabetic neuropathy. Tohoku J Exp Med. 2013;231(4):305-14.

45. Song CH, Petrofsky JS, Lee SW, Lee KJ, Yim JE. Effects of an exercise program on balance and trunk proprioception in older adults with diabetic neuropathies. Diabetes Technol Ther. 2011;13(8):803-11.

46. Taube W, Gruber M, Gollhofer A. Spinal and supraspinal adaptations associated with balance training and their functional relevance. Acta Physiol Oxf. 2008;193(2):101-16.

47. Zech A, Hübscher M, Vogt L, Banzer W, Hänsel F, Pfeifer K. Balance training for neuromuscular control and performance enhancement: a systematic review. J Athl Train. 2010;45(4):392-403.

48. Granacher U, Muehlbauer T, Taube W, Gollhofer A, Gruber M. Sensorimotor Training. In: Strength and conditioning: biological principles and practical applications. San Francisco: Wiley-Blackwell; 2011. p. 399.

49. Cnyrim C, Mergner T, Maurer C. Potential roles of force cues in human stance control. Exp Brain Res. 2009:194(3):419-33.

50. Maurer C, Mergner T, Peterka RJ. Multisensory control of human upright stance. Exp Brain Res. 2006;171(2):231-50.

51. Maurer C, Peterka RJ. A new interpretation of spontaneous sway measures based on a simple model of human postural control. J Neurophysiol. 2005 93(1):189-200

52. Prieto TE, Myklebust JB, Hoffmann RG, Lovett EG, Myklebust BM. Measures of postural steadiness: differences between healthy young and elderly adults. IEEE Trans Biomed Eng. 1996;43(9):956-66.

53. Peterka RJ. Sensorimotor integration in human postural control. J Neurophysiol. 2002;88(3):1097-118.

54. Wiesmeier IK, Dalin D, Wehrle A, Granacher U, Muehlbauer T, Dietterle J, et al. Balance training enhances vestibular function and reduces overactive proprioceptive feedback in elderly. Front Aging Neurosci. 2017;9:273.

55. van der Kooij H, Jacobs R, Koopman B, van der Helm F. An adaptive model of sensory integration in a dynamic environment applied to human stance control. Biol Cybern. 2001;84(2):103-15.

56. Mergner T, Maurer C, Peterka RJ. Sensory contributions to the control of stance: a posture control model. Adv Exp Med Biol. 2002;508:147-52.

57. Welch TDJ, Ting LH. A feedback model explains the differential scaling of human postural responses to perturbation acceleration and velocity. J Neurophysiol. 2009;101(6):3294-309.

58. Streckmann F, Kneis S, Leifert JA, Baumann FT, Kleber M, Ihorst G, et al. Exercise program improves therapy-related side-effects and quality of life in lymphoma patients undergoing therapy. Ann Oncol. 2014;25(2):493-9.

59. Boucher P, Teasdale N, Courtemanche R, Bard C, Fleury M. Postural stability in diabetic polyneuropathy. Diabetes Care. 1995;18(5):638-45.

60. Corriveau H, Prince F, Hébert R, Raîche M, Tessier D, Maheux P, et al. Evaluation of postural stability in elderly with diabetic neuropathy. Diabetes Care. 2000;23(8):1187-91.
61. Di Nardo W, Ghirlanda G, Cercone S, Pitocco D, Soponara C, Cosenza A, et al. The use of dynamic posturography to detect neurosensorial disorder in IDDM without clinical neuropathy. J Diabetes Complicat. 1999;13(2):79-85.

62. Simoneau GG, Ulbrecht JS, Derr JA, Becker MB, Cavanagh PR. Postural instability in patients with diabetic sensory neuropathy. Diabetes Care. 1994; 17(12):1411-21.

63. Uccioli L, Giacomini PG, Monticone G, Magrini A, Durola L, Bruno E, et al. Body sway in diabetic neuropathy. Diabetes Care. 1995;18(3):339-44.

64. van der Kooij H, Peterka RJ. Non-linear stimulus-response behavior of the human stance control system is predicted by optimization of a system with sensory and motor noise. J Comput Neurosci. 2011;30(3):759-78.

65. Horak FB, Dickstein R, Peterka RJ. Diabetic neuropathy and surface swayreferencing disrupt somatosensory information for postural stability in stance. Somatosens Mot Res. 2002;19(4):316-26.

66. Tucker MG, Kavanagh JJ, Barrett RS, Morrison S. Age-related differences in postural reaction time and coordination during voluntary sway movements. Hum Mov Sci. 2008;27(5):728-37.

67. Allum JHJ, Carpenter MG, Honegger F, Adkin AL, Bloem BR. Age-dependent variations in the directional sensitivity of balance corrections and compensatory arm movements in man. J Physiol. 2002;542(Pt 2):643-63.

68. Mergner T, Maurer C, Peterka RJ. A multisensory posture control model of human upright stance. Prog Brain Res. 2003;142:189-201.

69. Ormel HL, van der Schoot GGF, Sluiter WJ, Jalving M, Gietema JA, Walenkamp AME. Predictors of adherence to exercise interventions during and after cancer treatment: a systematic review. Psychooncology. 2018; 27(3):713-24.

70. Cata JP, Weng HR, Lee BN, Reuben JM, Dougherty PM. Clinical and experimental findings in humans and animals with chemotherapy-induced peripheral neuropathy. Minerva Anestesiol. 2006;72(3):151-69.

71. Brown JC, Harhay MO, Harhay MN. Physical function as a prognostic biomarker among cancer survivors. Br J Cancer. 2015;112(1):194-8.

\section{Publisher's Note}

Springer Nature remains neutral with regard to jurisdictional claims in published maps and institutional affiliations.

\section{Ready to submit your research? Choose BMC and benefit from:}

- fast, convenient online submission

- thorough peer review by experienced researchers in your field

- rapid publication on acceptance

- support for research data, including large and complex data types

- gold Open Access which fosters wider collaboration and increased citations

- maximum visibility for your research: over $100 \mathrm{M}$ website views per year

At $\mathrm{BMC}$, research is always in progress.

Learn more biomedcentral.com/submission 